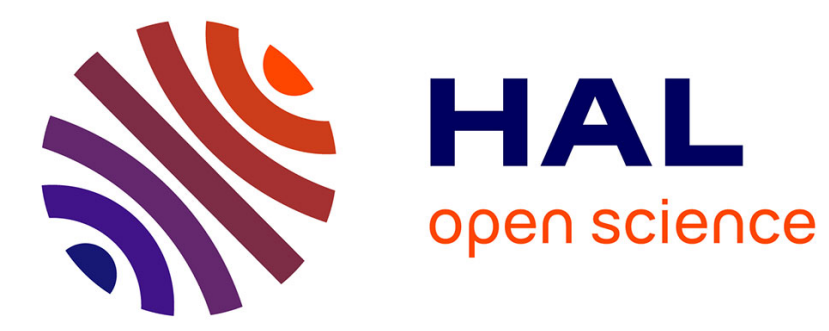

\title{
Decisions with conflicting and imprecise information
}

\author{
Thibault Gajdos, Jean-Christophe Vergnaud
}

\section{To cite this version:}

Thibault Gajdos, Jean-Christophe Vergnaud. Decisions with conflicting and imprecise information. Social Choice and Welfare, 2013, 41 (2), pp.427-452. 10.1007/s00355-012-0691-1 . hal-00443075

\section{HAL Id: hal-00443075 \\ https://hal.science/hal-00443075}

Submitted on 28 Dec 2009

HAL is a multi-disciplinary open access archive for the deposit and dissemination of scientific research documents, whether they are published or not. The documents may come from teaching and research institutions in France or abroad, or from public or private research centers.
L'archive ouverte pluridisciplinaire HAL, est destinée au dépôt et à la diffusion de documents scientifiques de niveau recherche, publiés ou non, émanant des établissements d'enseignement et de recherche français ou étrangers, des laboratoires publics ou privés. 


\section{ECOLE POLYTECHNIQUE}

CENTRE NATIONAL DE LA RECHERCHE SCIENTIFIQUE

DECISIONS WITH CONFLICTING AND IMPRECISE INFORMATION

Thibault GAJDOS

Jean-Christophe VERGNAUD

December 2009

Cahier $n^{\circ}$ 2009-57

\section{DEPARTEMENT D'ECONOMIE}

Route de Saclay

91128 PALAISEAU CEDEX

(33) 169333033

http://www.enseignement.polytechnique.fr/economie/

mailto:chantal.poujouly@polytechnique.edu 


\title{
Decisions with conflicting and imprecise information $^{1}$
}

\author{
Thibault Gajdos ${ }^{2} \quad$ Jean-Christophe Vergnaud ${ }^{3}$
}

December 2009

\footnotetext{
${ }^{1}$ We thank I. Gilboa, Ch. Gollier, N. Vieille, and audience at the Toulouse Shcool of Economics Theory seminar for useful comments and suggestions.

${ }^{2}$ CNRS, Ecole Polytechnique and CERSES. E-mail: gajdos@univ-paris1.fr.

${ }^{3}$ CNRS, Centre d'Economie de la Sorbonne and CERSES. E-mail: vergnaud@univ-paris1.fr.
} 


\begin{abstract}
The most usual procedure when facing decisions in complex settings consists in consulting experts, aggregating the information they provide, and deciding on the basis of this aggregated information. We argue that such a procedure entails a substantial loss, insofar as it precludes the possibility to take into account simultaneously the decision maker's attitude towards conflict among experts and her attitude towards imprecision of information. We propose to consider directly how a decision maker behaves when using information coming from several sources. We give an axiomatic foundation for a decision criterion that allows to distinguish on a behavioral basis the decision maker's attitude towards imprecision and towards conflict.
\end{abstract}

Keywords. Decisions with multiple sources of information. Conflict aversion. Imprecision aversion.

JEL Classification. D81, D83. 


\section{Introduction}

When facing situations involving uncertainty, a decision maker might seek the advice of experts. She will then make her decision on the basis of the information gathered. Such would be the case, for instance, of a company who consults experts in climate before deciding to invest in a ski resort. This raises the following question: how to decide on the basis of an information coming from several experts?

Clearly, different experts might have different opinions. Moreover, they could also provide imprecise information. We argue that one should take this two dimensions into account. Let us illustrate this point with the following example. Suppose that two experts in climatic change give their predictions about the frequency of snowy winters in the next thirty years. We allow the experts to express their degrees of beliefs through probability intervals. The size of the intervals captures the imprecision of their opinions. We compare now three possible situations, described in the table below.

\begin{tabular}{|l|c|c|}
\hline & Expert 1 & Expert 2 \\
\hline Situation A & $\frac{1}{2}$ & $\frac{1}{2}$ \\
\hline Situation B & $\frac{1}{4}$ & $\frac{3}{4}$ \\
\hline Situation C & {$\left[\frac{1}{4}, \frac{3}{4}\right]$} & {$\left[\frac{1}{4}, \frac{3}{4}\right]$} \\
\hline
\end{tabular}

Consider first situations A and B. A classical aggregation procedure is the linear aggregation rule ${ }^{1}$. According to this rule (and assuming that both experts are equally reliable), one would end in both situations with the same aggregated information, namely that the frequency of snowy winter in the next thirty years will be $\frac{1}{2}$. However, these two situations are rather different, as in the first one experts reach a consensus, whereas in the second one they strongly disagree. Thus, the aggregation procedure sweeps conflict under the rug.

A simple way out consists in aggregating experts opinions by probability intervals. A natural candidate in situation B would be the interval $\left[\frac{1}{4}, \frac{3}{4}\right]$. But consider now situation C. Admittedly, any sensible aggregation rule should respect unanimity among experts. Thus the aggregation in situation $\mathrm{C}$ should also lead to the interval $\left[\frac{1}{4}, \frac{3}{4}\right]$. However, situations $\mathrm{B}$ and $\mathrm{C}$ greatly differ.

\footnotetext{
${ }^{1}$ This aggregating rule, applied to probability distributions, is known in the statistics literature as the "pooling rule". See Stone (1961), McConway (1981), and Genest and Zidek (1986) for a survey.
} 
Indeed, in situation $\mathrm{B}$, experts provide strongly conflicting but precise information, whereas in situation $\mathrm{C}$, they provide strongly imprecise but similar predictions. The aggregation procedure considered does not allow to distinguish between imprecision of, and conflict among, experts.

This example suggests that it might be tricky to find an aggregation procedure that takes into account simultaneously, in a satisfactory way, both imprecision of experts assessments and conflict among them. This would not be a problem if decision makers were indifferent between ambiguity coming from imprecision of experts, or conflict among them. The few experimental studies that have addressed this question to date suggest that such is not the case. In particular, Smithson (1999) introduces the distinction between "source conflict" and "source ambiguity". He formulated the following "conflict aversion hypothesis":

Likewise, conflicting messages from two equally believable sources may be more disturbing in general than two informatively equivalent, ambiguous, but agreeing messages from the same sources.

Smithson (1999), p.184

Smithson finds evidence supporting this hypothesis in experiments with students involving verbal statements. Cabantous (2007) conducted experiments in a probabilistic setting (very similar to our example) with professional actuaries. She confirms the conflict aversion hypothesis, which means in this context that situation $\mathrm{C}$ is preferred to situation $\mathrm{B}$.

These results suggest that one should consider directly how a decision maker behaves when using information coming from several sources. However, to the best of our knowledge, there is no decision model that rationalizes the conflict aversion hypothesis. We axiomatically characterize preferences that exhibit independently aversion towards imprecision and conflict. We obtain a two-step procedure. The first step consists in using separately experts assessments in a multiple prior model. The second step consists in an aggregation of these evaluations through a multiple weights model. Such a model is compatible with the evidence found by Smithson (1999) and Cabantous (2007). More precisely, a decision maker will satisfy the conflict aversion hypothesis whenever her degree of conflict aversion is higher than her degree of imprecision aversion. 
The rest of the paper is organized as follows. We present the formal setup in Section 2. Section 3 is devoted to the axiomatic characterization of the decision maker preferences. We in particular introduce an axiom of conflict aversion. In Section 4 we present results on imprecision aversion and conflict aversion.

\section{Setup}

Let $\Omega$ be a finite set of states of the world. ${ }^{2}$ Let $\Delta(\Omega)$ be the set of all probability distributions over $\Omega$, and $\mathscr{P}$ be the family of compact and convex subsets of $\Delta(\Omega)$, where compactness is defined with regard to the Euclidean space $\mathbb{R}^{\Omega}$. The support of $P \in \mathscr{P}$, denoted $\operatorname{supp}(P)$, is defined as the union over $p \in P$ of the support of $p$.

The collection of information sets $\mathscr{P}$ is a mixture space under the operation defined by

$$
\lambda P+(1-\lambda) Q=\{\lambda p+(1-\lambda) q: p \in P, q \in Q\}
$$

The set of pure outcomes is denoted by $X$. Let $\Delta(X)$ be the set of simple lotteries (probability measures with finite supports) over $X$. Let $\mathscr{F}=\{f$ : $\Omega \rightarrow \Delta(X)\}$ be the set of acts. Abusing notation, any lottery is viewed as a constant act which delivers that lottery regardless of the states. The set $\mathscr{F}$ is a mixture space under the operation defined by:

$$
(\alpha f+(1-\alpha) g)(\omega)=\alpha f(\omega)+(1-\alpha) g(\omega), \forall \omega \in \Omega
$$

For $E \in \mathscr{S}$, denote $f_{E} g$ the act that yields $f(\omega)$ if $\omega \in E$ and $g(\omega)$ if not. We denote by $\mathscr{F}^{c}$ the set of constant acts, and for all $(\bar{x}, \underline{x}) \in X^{2}$, define $\mathscr{F}_{\bar{x}, \underline{x}}^{b}=$ $\left\{f \in \mathscr{F} \mid f(s)=\left(\bar{x}, p_{s} ; \underline{x}, 1-p_{s}\right), \forall s \in \Omega\right\}$, where $\left(\bar{x}, p_{s} ; \underline{x}, 1-p_{s}\right)$ denotes the lottery that yields $\bar{x}$ with probability $p_{s}$ and $\underline{x}$ with probability $\left(1-p_{s}\right)$.

The decision maker is endowed with a preference relation $\succcurlyeq$ defined on $\mathscr{F} \times \mathscr{P} \times \mathscr{P}$. When

$$
\left(f, P_{1}, P_{2}\right) \succcurlyeq\left(g, Q_{1}, Q_{2}\right)
$$

\footnotetext{
${ }^{2}$ The finiteness assumption is not needed, and is only made for sake of simplicity. All our results extend to the infinite countable case.
} 
the decision maker prefers choosing $f$ when, according to expert $i$, the true probability distribution belongs to $P_{i}(i \in\{1,2\})$ to choosing $g$ when, according to expert $i$, the true probability distribution belongs to $Q_{i}(i \in\{1,2\})$. The behavioral meaning of this relation has been extensively discussed in Gajdos, Hayashi, Tallon, and Vergnaud (2008), and we will not dwell on it here.

We derive from $\succcurlyeq$ a preference relation $\succcurlyeq^{*}$ on $\mathscr{F} \times \mathscr{P}$ as follows: $(f, P) \succcurlyeq^{*}$ $(g, Q)$ iff $(f, P, P) \succcurlyeq(g, Q, Q)$.

\section{Representation}

\subsection{Axioms}

Our axioms can be divided in two groups. The seven first axioms are simple extensions of the axioms used in Gajdos, Hayashi, Tallon, and Vergnaud (2008). They are used to derive a maxmin expected utility representation $\grave{a}$ la Gilboa and Schmeidler (1989), in presence of objective information set. Whereas Gajdos, Hayashi, Tallon, and Vergnaud (2008) only consider situations where one information set is available, we consider here an objective information made of a pair of probability distribution sets.

Axioms 1 to 6 exactly parallel Gilboa and Schmeidler (1989) axioms, so we will not elaborate on them. We only insist on the fact that imprecision aversion is captured through attitude towards mixture of acts (axiom 5). It is traditionally related to uncertainty aversion (hence its name; see in particular Schmeidler (1989) and Gilboa and Schmeidler (1989)). It turns out that in our context, it also can be interpreted as imprecision aversion, exactly through the same line of arguments. Axiom 7 parallels the classical (and crucial) cindependence axiom. It imposes that mixing all information sets with the same information set for both experts does not change preferences.

The two last axioms (Dominance and Conflict aversion) are specifically related to the problem at hand.

Axiom 1 (Order). The preference relation $\succcurlyeq$ is complete and transitive.

Axiom 2 (Continuity). For all $P, Q \in \mathscr{P}, f, g, h \in \mathscr{F}$, if $(f, P, Q) \succ(g, P, Q) \succ$ $(h, P, Q)$ then there exist $\alpha$ and $\beta$ in $(0,1)$ such that $(\alpha f+(1-\alpha) h, P, Q) \succ$ $(g, P, Q) \succ(\beta f+(1-\beta) h, P, Q)$. 
Axiom 3 (Risk preferences). (i) For all $f \in \mathscr{F}^{c}$ and $P, Q \in \mathscr{P},(f, P) \sim^{*}$ $(f, Q)$ and (ii) There exist $f, g \in \mathscr{F}, P \in \mathscr{P}$ such that $(f, P) \succ^{*}(g, P)$.

Axiom 4 (C-Independence). For all $f, g \in \mathscr{F}, h \in \mathscr{F}^{c}, P, Q \in \mathscr{P}, \alpha \in(0,1)$,

$$
(f, P, Q) \succcurlyeq(g, P, Q) \Rightarrow(\alpha f+(1-\alpha) h, P, Q) \succcurlyeq(\alpha g+(1-\alpha) h, P, Q) .
$$

Axiom 5 (Uncertainty aversion). For all $f, g \in \mathscr{F}, P \in \mathscr{P}, \alpha \in(0,1)$,

$$
(f, P) \sim^{*}(g, P) \Rightarrow(\alpha f+(1-\alpha) g, P) \succcurlyeq^{*}(f, P) .
$$

Axiom 6 (Monotonicity). For all $f, g \in \mathscr{F}, P \in \mathscr{P}$,

$$
(f(\omega), P) \succcurlyeq^{*}(g(\omega), P) \forall \omega \in \operatorname{supp}(P) \Rightarrow(f, P) \succcurlyeq^{*}(g, P) .
$$

Axiom 7 (I-Independence). For all $f \in \mathscr{F}, \alpha \in(0,1), P, Q, R \in \mathscr{P}$, if $(f, P) \succcurlyeq^{*}(f, Q)$ then

$$
(f, \alpha P+(1-\alpha) R) \succcurlyeq^{*}(g, \alpha Q+(1-\alpha) R) .
$$

The next two axioms are more specifically related to the problem at hand (namely, the aggregation of information). The first one states that if $(i)$ the decision maker prefers $f$ when both experts agree on an information set $P_{1}$ to $g$ when both experts agree on $Q_{1}$, and $(i i)$ she also prefers $f$ when both experts agree on an information set $P_{2}$ to $g$ when both experts agree on $Q_{2}$, then she prefers $f$ when experts' opinions are $\left(P_{1}, P_{2}\right)$ to $g$ when experts' opinions are $\left(Q_{1}, Q_{2}\right)$. This is essentially a dominance axiom, similar in substance to the traditional Pareto requirement.

Axiom 8 (Dominance). For all $f, g \in \mathscr{F}, P_{1}, Q_{1}, P_{2}, Q_{2} \in \mathscr{P}$,

$$
\left.\begin{array}{l}
\left(f, P_{1}\right) \succcurlyeq^{*}\left(g, Q_{1}\right) \\
\left(f, P_{2}\right) \succcurlyeq^{*}\left(g, Q_{2}\right)
\end{array}\right\} \Rightarrow\left(f, P_{1}, P_{2}\right) \succcurlyeq\left(g, Q_{1}, Q_{2}\right)
$$

Moreover, if one of the preferences on the left-hand side is strict, so is the preference on the right-hand side.

Our last axiom is related to conflict aversion. Given two information sets $P$, $Q$ and $\alpha \in(0,1)$, the information set $\alpha P+(1-\alpha) Q$ can be seen as a compromise 
between $P$ and $Q$ in the following sense. It is the set of probability distributions obtained if one considers that the true probability distribution belongs to $P$ with probability $\alpha$, whereas it belongs to $Q$ with probability $(1-\alpha)$. Our axiom says that the decision maker always prefers when experts come in with opinions that are less disparate, i.e., always prefers $(f, \alpha P+(1-\alpha) Q, \alpha Q+(1-\alpha) P)$ to $(f, P, Q)$.

Axiom 9 (Conflict aversion). For all $f \in \mathscr{F}, P, Q \in \mathscr{P}, \alpha \in(0,1)$,

$$
(f, \alpha P+(1-\alpha) Q, \alpha Q+(1-\alpha) P) \succcurlyeq(f, P, Q) .
$$

\subsection{Main result}

We derive from our axioms the following representation.

Theorem 1. Axioms 1 to 9 are satisfied iff there exist a mixture-linear function $u: \Delta(X) \rightarrow \mathbb{R}$, a linear mapping $\varphi: \mathscr{P} \rightarrow \mathscr{P}$ satisfying $\operatorname{supp}(\varphi(P)) \subseteq$ $\operatorname{supp}(P)$ and a symmetric closed and convex subset $\Pi$ in $\Delta(\{1,2\})$ such that $\succcurlyeq$ can be represented by:

$V(f, P, Q)=\min _{\pi \in \Pi} \pi(1)\left(\min _{p \in \varphi(P)} \sum_{\omega} u(f(\omega)) p(\omega)\right)+\pi(2)\left(\min _{p \in \varphi(Q)} \sum_{\omega} u(f(\omega)) p(\omega)\right)$.

Moreover $u$ is unique up to a positive linear transformation, and $\varphi$ and $\Pi$ are unique.

Maximizing this formula can be though of as a two-step procedure. First, the decision maker transforms experts information through $\varphi$, and uses the resulting sets of probability to evaluate the act under consideration. Second, she aggregates linearly these two evaluations, using the worst weight vector in a set $\Pi$. The first step deals with experts' assessments. It is important to observe that we face two very different kinds of sets of probability distributions. Indeed, $P$ and $Q$ are strictly informational. They capture the information available to the decision maker. $\varphi(P)$ and $\varphi(Q)$ are the behavioral beliefs the decision maker would use to evaluate acts if she were facing either $P$ or $Q^{3}$. The mapping $\varphi$ introduces a subjective treatment of an imprecise information.

\footnotetext{
${ }^{3}$ In Gilboa and Schmeidler (1989) celebrated maxmin expected utility model, only behavioral beliefs appear.
} 
In the second step, evaluations based on information provided by experts are aggregated. The set $\Pi$ captures the decision maker's attitude towards conflict among valuations based on experts assessments.

Note that we also have:

$$
V(f, P, Q)=\min _{p \in \Pi \otimes(\varphi(P), \varphi(Q))} \sum_{\omega} u(f(\omega)) p(\omega)
$$

where $\Pi \otimes(\varphi(P), \varphi(Q))=\{\pi(1) p+\pi(2) q \mid \pi \in \Pi, p \in \varphi(P), q \in \varphi(Q)\}$. Thus maximizing this formula can be though of as first aggregating experts information through $\Pi \otimes(\varphi(P), \varphi(Q))$, and then applying the maxmin expected utility criterion over this set. Therefore $\Pi \otimes(\varphi(P), \varphi(Q))$ has only a behavioral meaning: it is related to the decision maker's preferences.

Finally, note that a different route is also possible: It consists in aggregating behavioral beliefs of experts into behavioral beliefs of the decision maker. It essentially amounts to ask the experts what their own decision would be (possibly assuming that they would evaluate consequences the same way as the decision maker) and to aggregate these stated preferences. A large literature in social choice theory has been elaborated along these lines, following Harsanyi (1955) seminal paper ${ }^{4}$. See among others Mongin (1995), Gilboa, Samet, and Schmeidler (2004), Gajdos, Tallon, and Vergnaud (2008).

Indeed, this problem has also been addressed by Crès, Gilboa, and Vieille (2009), who consider the problem of aggregating preferences of Maxmin Expected Utility maximizers who share the same utility function into a Maxmin Expected utility. They show that under Pareto constraint, the aggregate set of priors takes the form $\Pi \otimes\left(P_{1}, \ldots, P_{n}\right)$, where $P_{i}$ denotes individual $i$ 's set of priors. They thus aggregate behavioral beliefs into behavioral beliefs. ${ }^{5}$

\footnotetext{
${ }^{4}$ Although Harsanyi (1955) actually considers the case where experts share the same beliefs, and disagree on valuations. But it really laid the foundations for the aggregation of experts behavioral beliefs.

${ }^{5}$ Actually, one can also interpret experts beliefs in Crès, Gilboa, and Vieille (2009) as informational beliefs. But then it implies that the decision maker is forced to be extremely averse towards uncertainty.
} 


\section{Attitude towards uncertainty and conflict}

\subsection{General definitions and characterization}

We now turn to the behavioral characterization of imprecision and conflict aversion. We use the standard comparative approach.

We first define comparative imprecision aversion as in Gajdos, Tallon, and Vergnaud (2004) and Gajdos, Hayashi, Tallon, and Vergnaud (2008). Let a and $b$ be two decision makers. We will say that $b$ is more averse to imprecision than $a$ if, whenever $a$ prefers a precise situation to an imprecise one, so does $b$. In order to control for risk aversion, this definition is restricted to binary acts.

Definition 1. Let $\succsim_{a}^{*}$ and $\succsim_{b}^{*}$ be two preference relations defined on $\mathscr{F} \times \mathscr{P}$. Suppose there exist two prizes $\bar{x}, \underline{x}$ in $X$ such that both a and $b$ strictly prefer $\bar{x}$ to $\underline{x}$. We say that $\succcurlyeq_{b}^{*}$ is more averse to imprecision than $\succcurlyeq_{a}^{*}$ whenever for all $f \in \mathscr{F}_{\bar{x}, \underline{x}}^{b}, p \in \Delta(\Omega), P \in \mathscr{P}$

$$
(f,\{p\}) \succcurlyeq_{a}^{*}(f, P) \Rightarrow(f,\{p\}) \succcurlyeq_{b}^{*}(f, P) .
$$

The following proposition shows how $\varphi$ is related to the decision maker's attitude towards imprecision. Intuitively, the more $\varphi$ "shrinks" $P$, the less imprecision averse is the decision maker.

Proposition 1. The following assertions are equivalent:

1. $\succcurlyeq_{b}^{*}$ is more averse to imprecision than $\succcurlyeq_{a}^{*}$,

2. for all $P \in \mathscr{P}, \varphi_{a}(P) \subset \varphi_{b}(P)$.

Comparative conflict aversion will be defined along the same line as comparative imprecision aversion. It simply states that decision maker $b$ is more averse to conflict than decision maker $a$ if, whenever $a$ prefers a consensual situation to a situation with divergent information, so does $b$.

Assume that a decision maker strictly prefers $(f, P, P)$ to $(f, Q, Q)$. Then (by axiom 8) she will have the following preferences: $(f, P, P) \succcurlyeq(f, P, Q) \succcurlyeq$ $(f, Q, Q)$. Now, consider $R(\alpha)=\alpha P+(1-\alpha) Q$. Of course, the larger $\alpha$, the better $(f, R(\alpha), R(\alpha))$. Since $(f, R(1), R(1)) \succcurlyeq(f, P, Q) \succcurlyeq(f, R(0), R(0))$, there is an (unique) $\hat{\alpha}$ such that $(f, R(\hat{\alpha}), R(\hat{\alpha})) \sim(f, P, Q)$. Loosely speaking, 
$R(\hat{\alpha})$ is the worst consensual information that the decision maker considers as equivalent to $(P, Q)$ when facing act $f$. Thus $(1-\hat{\alpha})$ can be seen as the "price" she is ready to pay to avoid conflict. Decision maker $b$ is more averse to conflict than decision maker $a$ if $b$ is ready to "pay" a higher price to avoid conflict.

Definition 2. Let $\succsim_{a}$ and $\succsim_{b}$ be two preference relations defined on $\mathscr{F} \times \mathscr{P} \times$ $\mathscr{P}$. We say that $\succsim_{b}$ is more averse to conflict than $\succsim_{a}$ if for all $f \in \mathscr{F}$, $P, Q \in \mathscr{P}, \alpha \in(0,1)$, such that both $a$ and $b$ prefers $(f, P)$ to $(f, Q)$ if :

$$
(f, \alpha P+(1-\alpha) Q, \alpha P+(1-\alpha) Q) \succcurlyeq_{a}(f, P, Q)
$$

then,

$$
(f, \alpha P+(1-\alpha) Q, \alpha P+(1-\alpha) Q) \succcurlyeq_{b}(f, P, Q) .
$$

The following Proposition shows how $\Pi$ is related to the decision maker's attitude towards conflict. Intuitively, the larger $\Pi$, the more averse to conflict is the decision maker.

Proposition 2. The following assertions are equivalent:

1. $\succcurlyeq_{b}$ is more averse to conflict than $\succcurlyeq_{a}$,

2. $\Pi_{a} \subseteq \Pi_{b}$.

\subsection{The two states case}

In order to illustrate our results, let us consider the specific situation where there are only two states, and $\varphi$ is symmetric, i.e., for all permutation $\rho$ : $\Omega \rightarrow \Omega$, and all $P \in \mathscr{P} \rho\left((\varphi(P))=\varphi\left(P_{\rho}\right)\right.$, where $\rho(\varphi(P))=\{p \mid \exists q \in$ $\varphi(P), q(\rho(\omega))=p(\omega), \forall \omega \in \Omega\}$ and $P_{\rho}=\{p \mid \exists q \in P, q(\rho(\omega))=p(\omega), \forall \omega \in$ $\Omega\}$.

In this case, $\varphi$ and $\Pi$ take the following parametric form:

$$
\varphi(P)=\{(1-\theta) c(P)+\theta p \mid p \in P\}
$$

where $\theta \in[0,1]$ and $c(P)$ is the center of $P$, and

$$
\Pi=\left\{(1-\alpha)\left(\frac{1}{2}, \frac{1}{2}\right)+\alpha(t, 1-t) \mid t \in[0,1]\right\},
$$


where $\alpha \in[0,1]$. In other words, $\varphi(P)$ is a contraction of $P$ around its center, with a contraction rate equal to $(1-\theta)$, whereas $\Pi$ is a symmetric set of probabilities. In view of Propositions 1 and 2, $\theta$ can be interpreted as a measure of imprecision aversion (imprecision aversion increases with $\theta$ ) whereas $\alpha$ can be interpreted as a measure of conflict aversion (conflict aversion increases with $\alpha)$.

The traditional aggregation approach assumes that decisions with information coming from various experts can be made in two steps. First, information provided by experts is somehow aggregated into a unique piece of information; then this aggregated information is used by the decision maker, who transforms it into behavioral beliefs. A classical example of this kind of decision process is the traditional cost-benefit analysis. Expert commission transmits the information concerning the likelihood of the relevant events, outcomes are evaluated separately, and the policy maker uses a decision rule based on transmitted information and outcome evaluation. This is typically the case for environmental and health issues (e.g., global warming, new diseases). The problem can then be reduced to independent questions. First, how should we aggregate experts opinions? Second, what should be the decision, given this aggregated information?

It is natural to wonder whether it is always possible to reduce the behavioral approach we follow to the traditional two-steps aggregation? This would be the case if any pair of statements is equivalent for the decision maker (from the informational point of view) to some statement coming from a single source. In other words, for all $P, Q$, there should exist $R$ such that for all $f,(f, P, Q) \sim$ $(f, R, R)$. As shown by the following proposition, such is actually not the case. When there are only two states and $\varphi$ is symmetric, the classical procedure and our approach are equivalent iff the decision maker degree of uncertainty aversion is larger than her degree of conflict aversion. Note that in these situations, the conflict aversion hypothesis described and observed by Smithson (1999) and Cabantous (2007) will not be satisfied.

Proposition 3. Assume $|\Omega|=2$ and $\varphi(P)$ is symmetric. Then the following statements are equivalent:

(i) For all $P, Q \in \mathscr{P}$, there exists $R \in \mathscr{P}$ such that $\Pi \otimes(\varphi(P), \varphi(Q))=$ $\varphi(R)$; 
(ii) $\theta \geq \alpha$.

In some specific cases we can even be more specific. For instance, if $Q \subseteq P$, there always exists $R$ such that $\varphi(R)=\Pi \otimes(\varphi(P), \varphi(Q))$ as soon as $\theta>0 . R$ is then simply:

$$
R=\left(\frac{1+\alpha}{2}\right) P+\left(\frac{1-\alpha}{2}\right) Q
$$

The example discussed in the introduction suggested that it might be difficult to take into account simultaneously the decision maker attitude towards imprecision and conflict when using a two-steps aggregation procedure. Indeed, it must then be the case that attitude towards conflict only appears in the first step, whereas attitude towards imprecision only appears in the second step. This would not be a problem if the two steps commute (i.e. if the traditional procedure were equivalent to first transforming experts informational beliefs into several behavioral beliefs, and then aggregating these behavioral beliefs). But, as shown by the following proposition, such is the case only if the decision maker is extremely averse to imprecision. Although this result is only proved with two states and a symmetric $\varphi$, it suggests that the behavioral approach we followed is significantly wider than the traditional one.

Proposition 4. Assume $|\Omega|=2$ and $\varphi(P)$ is symmetric. Then the following statements are equivalent:

(i) For all $P, Q \in \mathscr{P}, \Pi \otimes(\varphi(P), \varphi(Q))=\varphi(\Pi \otimes(P, Q))$;

(ii) $\theta=1$.

Finally, we suggested in the introduction that a natural way to aggregate information so as to take into account the decision maker attitude towards conflict consists in considering the unions of the pieces of information provided by the experts. It is thus a natural question to ask if this rule can be obtained in our framework. The answer is: yes. It actually corresponds to the case of extreme aversion towards both conflict and imprecision.

Proposition 5. Assume $|\Omega|=2$ and $\varphi(P)$ is symmetric. Then the following statements are equivalent:

(i) For all $P, Q \in \mathscr{P}, \Pi \otimes(\varphi(P), \varphi(Q))=\varphi(\operatorname{co}(P \cup Q))$;

(ii) $(\alpha, \theta)=(1,1)$. 
A

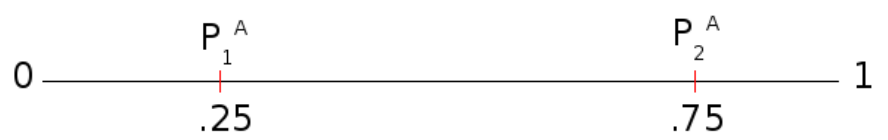

B $0 \longrightarrow P_{1}^{B}=P_{2}^{B}$
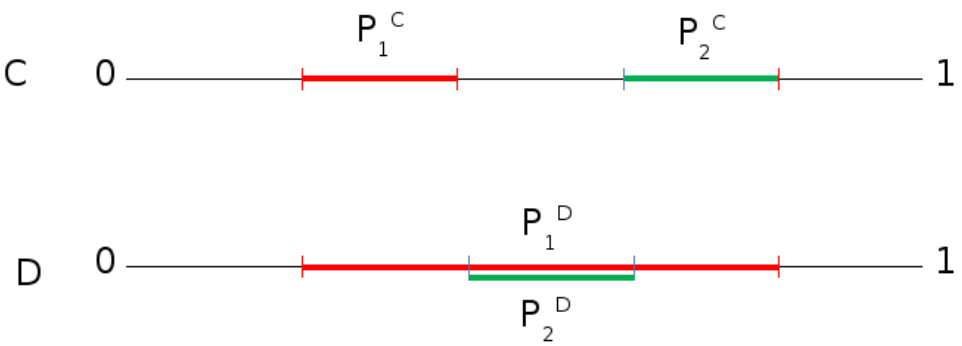

\section{Illustration}

We illustrate our approach through the following example. Assume $\Omega=\{1,2\}$. Consider the four following situations.
A. $P_{1}^{A}=\left\{\frac{1}{4}\right\}$ and $P_{2}^{A}=\left\{\frac{3}{4}\right\}$;
B. $P_{1}^{B}=P_{2}^{B}=\left\{p \mid \frac{1}{4} \leq p(1) \leq \frac{3}{4}\right\}$;
C. $P_{1}^{C}=\left\{p \mid \frac{1}{4} \leq p(1) \leq \frac{3}{8}\right\}$ and $P_{2}^{C}=\left\{p \mid \frac{5}{8} \leq p(1) \leq \frac{3}{4}\right\}$;
D. $P_{1}^{D}=P_{1}^{B}$ and $P_{2}^{D}=\left\{p \mid \frac{3}{8} \leq p(1) \leq \frac{5}{8}\right\}$.

These four situations are depicted on the following graph, where the range of $p(1)$ is depicted.

We assume that $\varphi$ and $\Pi$ are defined as in Section 4 . Let $\Psi\left(P_{1}^{i}, P_{2}^{i}\right)=$ $\Pi \otimes\left(\varphi\left(P_{1}^{i}\right), \varphi\left(P_{2}^{i}\right)\right)(i \in\{A, B, C, D\})$. Simple computations lead to:
A. $\Psi\left(P_{1}^{A}, P_{2}^{A}\right)=\left\{p \mid \frac{2-\alpha}{4} \leq p(1) \leq \frac{2+\alpha}{4}\right\}$;
B. $\Psi\left(P_{1}^{B}, P_{2}^{B}\right)=\left\{p \mid \frac{1-\theta}{4} \leq p(1) \leq \frac{2+\theta}{4}\right\}$;
C. $\Psi\left(P_{1}^{C}, P_{2}^{C}\right)=\left\{p \mid \frac{8-3 \alpha-\theta}{16} \leq p(1) \leq \frac{8+3 \alpha+\theta}{16}\right\}$; 
D. $\Psi\left(P_{1}^{D}, P_{2}^{D}\right)=\left\{p \mid \frac{5-\alpha \theta+3(1-\theta)}{16} \leq p(1) \leq \frac{11+\alpha \theta-3(1-\theta)}{16}\right\}$.

Clearly, if $\Psi\left(P_{1}^{i}, P_{2}^{i}\right) \subset \Psi\left(P_{1}^{j}, P_{2}^{j}\right)$, the decision maker prefers the situation ${ }^{6}$ where the available information is $\left(P_{1}^{i}, P_{2}^{i}\right)$ to the situation where the available information is $\left(P_{1}^{j}, P_{2}^{j}\right)$. The following table summarize the results. Situations are listed from the best to the worst, for different values of the parameters.

\begin{tabular}{|c|c|c|c|}
\hline $1 \geq \theta \geq \frac{3 \alpha}{\alpha+2}$ & $\frac{3 \alpha}{\alpha+2} \geq \theta \geq \frac{4 \alpha}{3+\alpha}$ & $\frac{4 \alpha}{3+\alpha} \geq \theta \geq \alpha$ & $\alpha \geq \theta \geq 0$ \\
\hline$A$ & $A$ & $D$ & $D$ \\
$C$ & $D$ & $A$ & $B$ \\
$D$ & $C$ & $C$ & $C$ \\
$B$ & $B$ & $B$ & $A$ \\
\hline
\end{tabular}

Note that increasing imprecision aversion implies moving from the right to the left of the table, whereas increasing conflict aversion implies moving from the left to the right. Thus, as imprecision aversion increases (ceteris paribus) $A$ and $C$ are ranked higher, and $B$ and $D$ lower. The converse is true when conflict aversion increases. Observe that the conflict aversion hypothesis corresponds to the situation where situation $B$ is better than situation $A$, i.e., the last column. Unsurprisingly, it is obtained when the degree of conflict aversion is higher than the degree of imprecision aversion.

\section{Appendix}

\subsection{Proof of Theorem 1}

Necessity is easily checked. We thus only prove sufficiency.

By Proposition 2 in Gajdos, Hayashi, Tallon, and Vergnaud (2008), we have:

Lemma 1. If axioms 1 to 7 hold, then there exist a function $U: \mathscr{F} \times \mathscr{P} \rightarrow$ $\mathbb{R}$ which represents $\succcurlyeq^{*}$, a mixture-linear function $u: \Delta(X) \rightarrow \mathbb{R}$, a linear mapping $\varphi: \mathscr{P} \rightarrow \mathscr{P}$ satisfying $\operatorname{supp}(\varphi(P)) \subseteq \operatorname{supp}(P)$ such that:

$$
U(f, P)=\min _{p \in \varphi(P)} E_{p} u(f)
$$

\footnotetext{
${ }^{6}$ We say that a decision maker prefers a situation $(P, Q)$ to a situation $\left(P^{\prime}, Q^{\prime}\right)$ when for any bet, she prefers betting in situation $(P, Q)$ rather than in situation $\left(P^{\prime}, Q^{\prime}\right)$.
} 
Moreover $u$ is unique up to a positive linear transformation, and $\varphi$ is unique.

According to Lemma 1, $u$ is unique up to a positive linear transformation, which implies that we can choose $u$ such that $U\left(h_{1}, P\right)=1$ and $U\left(h_{2}, P\right)=-1$ for some constant acts $h_{1}, h_{2}$, and for any $P$.

Lemma 2. $\{(U(f, P),(f, Q)) \mid f \in \mathscr{F}, P, Q \in \mathscr{P}\}=$ range $U \times$ range $U$.

Proof. Let $K=\{(U(f, P), U(f, Q)) \mid f \in \mathscr{F}, P, Q \in \mathscr{P}\}$, and $\mathscr{D}=$ range $U \times$ range $U$. We have to show that $\mathscr{D} \subseteq K$. Let $f, g \in \mathscr{F}$ and $P, Q \in \mathscr{P}$. By axiom 6 there exist $\bar{f}$ and $\underline{f}$ in $\mathscr{F}$ such that $(\bar{f}, P) \succcurlyeq^{*}(f, P) \succcurlyeq^{*}(\underline{f}, P)$. Thus, by axiom 2 there exists $\theta \in[0,1]$ such that $(\theta \bar{f}+(1-\theta) \underline{f}, P) \sim^{*}(f, P)$. Let $f^{c}=\theta \bar{f}+(1-\theta) \underline{f}$. Observe that $f^{c} \in \mathscr{F}^{c}$ and $U(f, P)=U\left(f^{c}, P\right)$. Define similarly $g^{c}$ as the constant act such that $\left(g^{c}, Q\right) \sim(g, Q)$. We also have, of course, $U(g, Q)=U\left(g^{c}, Q\right)$. Now, fix any event $E \subset \Omega, E \neq \Omega$, and let $P^{\prime}, Q^{\prime} \in$ $\mathscr{P}$ be such that $\operatorname{supp}\left(P^{\prime}\right) \subseteq E$ and $\operatorname{supp}\left(Q^{\prime}\right) \subseteq \Omega \backslash E$. We have $U\left(f_{E}^{c} g^{c}, P^{\prime}\right)=$ $U\left(f^{c}, P\right)$ by axioms 3 and 6 . Thus $U\left(f_{E}^{c} g^{c}, P^{\prime}\right)=U(f, P)$. Similarly, we have $U\left(f_{E}^{c} g^{c}, Q^{\prime}\right)=U\left(g^{c}, Q^{\prime}\right)=U(g, Q)$. Thus $(U(f, P), U(g, Q)) \in K$, the desired result.

By axioms 1 and 2, there exists a continuous function $V: \mathscr{F} \times \mathscr{P} \times \mathscr{P} \rightarrow \mathbb{R}$ that represents $\succcurlyeq$. By axiom 8, and Lemma 2 there exists an increasing, continuous function $\tilde{V}: \mathscr{D} \rightarrow \mathbb{R}$ such that for all $f \in \mathscr{F}$ and $P, Q \in \mathscr{P}$ :

$$
V(f, P, Q)=\tilde{V}(U(f, P), U(f, Q))
$$

Moreover, given $U, V$ can be chosen such that $V(f, P, P)=U(f, P)$ (since $V(f, P, P)$ represents $\succcurlyeq^{*}$ as well). The two following steps essentially mimic Gilboa and Schmeidler (1989)'s and Chateauneuf (1991) proofs.

Lemma 3. For all $w \in \mathscr{D}, \alpha \in[0,1]$ such that $\alpha w \in \mathscr{D}, \tilde{V}(\alpha w)=\alpha \tilde{V}(w)$.

Proof. Pick $f_{0} \in \mathscr{F}^{c}$ such that $U\left(f_{0}, P\right)=0$ (this is possible, given the normalization we choose for $V)$. Let $w=\left(w_{1}, w_{2}\right) \in \mathscr{D}$. By Lemma 2 there exist $f \in \mathscr{F}, Q_{1}, Q_{2} \in \mathscr{P}$ such that $U\left(f, Q_{1}\right)=w_{1}$ and $U\left(f, Q_{2}\right)=w_{2}$. By definition of $\tilde{V}$ and $V$ we have $V\left(f, Q_{1}, Q_{2}\right)=\tilde{V}(w)$. By axioms 2, 3 and 6 there exists $h \in \mathscr{F}^{c}$ such that $\left(h, Q_{1}, Q_{2}\right) \sim\left(f, Q_{1}, Q_{2}\right)$. By axiom 4 , 
$\left(\alpha h+(1-\alpha) f_{0}, Q_{1}, Q_{2}\right) \sim\left(\alpha f+(1-\alpha) f_{0}, Q_{1}, Q_{2}\right)$ for all $\alpha \in[0,1]$. By axiom $3,\left(\alpha h+(1-\alpha) f_{0}, Q_{1}, Q_{2}\right) \sim\left(\alpha h+(1-\alpha) f_{0}, Q_{1}, Q_{1}\right)$. Thus

$$
\begin{aligned}
V\left(\alpha h+(1-\alpha) f_{0}, Q_{1}, Q_{2}\right) & =V\left(\alpha h+(1-\alpha) f_{0}, Q_{1}, Q_{1}\right) \\
& =U\left(\alpha h+(1-\alpha) f_{0}, Q_{1}\right), \text { by the normalization of } V \\
& =\alpha U\left(h, Q_{1}\right)+(1-\alpha) U\left(f_{0}, Q_{1}\right) \text { by } c \text {-linearity of } U \\
& =\alpha U\left(h, Q_{1}\right), \text { since } U\left(f_{0}, Q_{1}\right)=0 \\
& =\alpha V\left(h, Q_{1}, Q_{1}\right), \text { by the normalization of } V \\
& =\alpha V\left(h, Q_{1}, Q_{2}\right), \text { by axiom } 3 \\
& =\alpha V\left(f, Q_{1}, Q_{2}\right), \text { by definition of } h \\
& =\alpha \tilde{V}(w) .
\end{aligned}
$$

On the other hand,

$$
\begin{aligned}
& V\left(\alpha f+(1-\alpha) f_{0}, Q_{1}, Q_{2}\right) \\
= & \tilde{V}\left(U\left(\alpha f+(1-\alpha) f_{0}, Q_{1}\right), U\left(\alpha f+(1-\alpha) f_{0}, Q_{2}\right)\right) \\
= & \tilde{V}\left(\alpha U\left(f, Q_{1}\right)+(1-\alpha) U\left(f_{0}, Q_{1}\right), \alpha U\left(f, Q_{2}\right)+(1-\alpha) U\left(f_{0}, Q_{2}\right)\right), \\
& \text { by } c \text {-linearity of } U \\
= & \tilde{V}\left(\alpha U\left(f, Q_{1}\right), \alpha U\left(f, Q_{2}\right)\right) \text { since } U\left(f_{0}, P\right)=0 \\
= & \tilde{V}(\alpha w) .
\end{aligned}
$$

Thus $\tilde{V}(\alpha w)=\alpha \tilde{V}(w)$ for all $\alpha \in[0,1]$, and thus for all $\alpha \in \mathbb{R}_{+}$.

We extend $\tilde{V}$ to $\mathbb{R}^{2}$ by homogeneity, and still call $\tilde{V}$ its extension (which is homogeneous and monotone).

Lemma 4. For all $w \in \mathbb{R}^{2}, \mu \in \mathbb{R}, \tilde{V}(w+(\mu, \mu))=\tilde{V}(w)+\mu$.

Proof. Let $w_{1}, w_{2}, \mu$ be such that $2 w_{1}, 2 w_{2}, 2 \mu \in$ range $U$. Given the homogeneity of $\tilde{V}$, this assumption is without any loss of generality. Let $f \in \mathscr{F}$, $h_{1}, h_{2} \in \mathscr{F}^{c}$ and $P, Q \in \mathscr{P}$ be such that $U(f, P)=2 w_{1}, U(f, Q)=2 w_{2}$, $\left(h_{1}, P, Q\right) \sim(f, P, Q)$ and $U\left(h_{2}, P\right)=2 \mu$. Since $\left(h_{1}, P, Q\right) \sim(f, P, Q)$, axiom 4 implies $\left(\frac{1}{2} h_{1}+\frac{1}{2} h_{2}, P, Q\right) \sim\left(\frac{1}{2} f+\frac{1}{2} h_{2}, P, Q\right)$. Thus:

$$
\tilde{V}\left(U\left(\frac{1}{2} h_{1}+\frac{1}{2} h_{2}, P\right), U\left(\frac{1}{2} h_{1}+\frac{1}{2} h_{2}, Q\right)\right)=\tilde{V}\left(U\left(\frac{1}{2} f+\frac{1}{2} h_{2}, P\right), U\left(\frac{1}{2} f+\frac{1}{2} h_{2}, Q\right)\right),
$$


which implies

$$
U\left(\frac{1}{2} h_{1}+\frac{1}{2} h_{2}, P\right)=\tilde{V}\left(\frac{1}{2} U(f, P)+\frac{1}{2} U\left(h_{2}, P\right), \frac{1}{2} U(f, Q)+\frac{1}{2} U\left(h_{2}, Q\right)\right) .
$$

Therefore,

$$
\begin{aligned}
\tilde{V}\left(w_{1}+\mu, w_{2}+\mu\right) & =\frac{1}{2} U\left(h_{1}, P\right)+\frac{1}{2} U\left(h_{2}, P\right) \\
& =\frac{1}{2} \tilde{V}\left(U\left(h_{1}, P\right), U\left(h_{1}, Q\right)\right)+\mu, \text { by homogeneity of } U \\
& =\frac{1}{2} V\left(h_{1}, P, Q\right)+\mu, \text { by normalization of } V \\
& =\frac{1}{2} V(f, P, Q)+\mu \\
& =\frac{1}{2} \tilde{V}(U(f, P), U(f, Q))+\mu \\
& =\frac{1}{2} \tilde{V}\left(2 w_{1}, 2 w_{2}\right)+\mu \\
& =\tilde{V}\left(w_{1}, w_{2}\right)+\mu,
\end{aligned}
$$

the desired result.

It remains to show that $\tilde{V}$ is symmetric and concave. This is the key part of the proof, where the conflict aversion axiom plays a crucial role.

Lemma 5. $\tilde{V}$ is symmetric.

Proof. We first show that $\tilde{V}$ is symmetric. Without loss of generality (because of the homogeneity of $\tilde{V}$ ), choose $w, w^{\prime} \in \mathscr{D}$. Let $f \in \mathscr{F}$ and $P, Q \in \mathscr{P}$ be such that $U(f, P)=w_{1}$ and $U(f, Q)=w_{2}$. Let $\alpha \in[0,1]$. We have:

$$
\begin{aligned}
\tilde{V}\left(\alpha w_{1}\right. & \left.+(1-\alpha) w_{2}, \alpha w_{2}+(1-\alpha) w_{1}\right) \\
& =\tilde{V}(\alpha U(f, P)+(1-\alpha) U(f, Q), \alpha U(f, Q)+(1-\alpha) U(f, P)) \\
& =\tilde{V}(U(f, \alpha P+(1-\alpha) Q), U(\alpha Q+(1-\alpha) P)) \text {, by linearity of } \varphi .
\end{aligned}
$$

Thus axiom 9 implies for all $w_{1}, w_{2} \in \mathbb{R}$ (by homogeneity) and all $\alpha \in[0,1]$ :

$$
\tilde{V}\left(\alpha w_{1}+(1-\alpha) w_{2}, \alpha w_{2}+(1-\alpha) w_{1}\right) \geq \tilde{V}\left(w_{1}, w_{2}\right) .
$$


Thus, in particular, $\tilde{V}$ must be symmetric. Indeed, setting $\alpha=0$, we obtain $\tilde{V}\left(w_{2}, w_{1}\right) \geq \tilde{V}\left(w_{1}, w_{2}\right)$. Permuting $w_{1}$ and $w_{2}$ yields $\tilde{V}\left(w_{1}, w_{2}\right) \geq \tilde{V}\left(w_{2}, w_{2}\right)$, and thus $\tilde{V}\left(w_{1}, w_{2}\right)=\tilde{V}\left(w_{2}, w_{1}\right)$.

Lemma 6. $\tilde{V}$ is concave.

Proof. Step 1.

Let $w=\left(w_{1}, w_{2}\right)$ and $w^{\prime}=\left(w_{1}^{\prime}, w_{2}^{\prime}\right)$ in $\mathscr{D}$ be such that $\tilde{V}(w)=\tilde{V}\left(w^{\prime}\right)$, $w_{1} \leq w_{2}, w_{1}^{\prime} \leq w_{2}^{\prime}$, and $\bar{w}=(t, t) \in \mathscr{D}$ be such that $\tilde{V}(\bar{w})=\tilde{V}(w)$ (by axioms 2 and 8 , such an $\bar{w}$ exists). Without loss of generality, assume $0<w_{1} \leq w_{1}^{\prime}$ (and thus, by axiom $8, w_{2} \geq w_{2}^{\prime}$ ) and $0<w_{2}^{\prime}$. First, we show that there exists $\theta \in[0,1]$ such that $w^{\prime}=\theta w+(1-\theta) \bar{w}$. Assume that such is not the case. Let $\lambda>0$ be such that $\lambda w^{\prime} \in[w, \bar{w}]$, and let $\hat{\theta}$ be such that $\lambda w^{\prime}=\hat{\theta} w+(1-\hat{\theta}) \bar{w}$. Since $w^{\prime} \notin[w, \bar{w}], \lambda \neq 1$. Thus, by axiom 8 , either $\tilde{V}\left(\lambda w^{\prime}\right)>\tilde{V}\left(w^{\prime}\right)$ or $\tilde{V}\left(\lambda w^{\prime}\right)<\tilde{V}\left(w^{\prime}\right)$. But by lemma 3 and $4, \tilde{V}\left(\lambda w^{\prime}\right)=\tilde{V}(\hat{\theta} w+(1-\hat{\theta}) \bar{w})=$ $\theta \tilde{V}(w)+(1-\theta) \tilde{V}(\bar{w})=\tilde{V}(w)=\tilde{V}\left(w^{\prime}\right)$, a contradiction.

Thus, let $\theta$ be such that $w^{\prime}=\theta w+(1-\theta) \bar{w}$. Then, for all $\alpha \in[0,1]$,

$$
\begin{aligned}
\tilde{V}\left(\alpha w+(1-\alpha) w^{\prime}\right) & =\tilde{V}(\alpha w+(1-\alpha)(\theta w+(1-\theta) \bar{w})) \\
& =\tilde{V}((\alpha+(1-\alpha) \theta) w+(1-\alpha)(1-\theta) \bar{w}) \\
& =(\alpha+(1-\alpha) \theta) \tilde{V}(w)+(1-\alpha)(1-\theta) \tilde{V}(\bar{w}) \\
& =\tilde{V}(w) \\
& =\alpha \tilde{V}(w)+(1-\alpha) \tilde{V}\left(w^{\prime}\right),
\end{aligned}
$$

where the third equality follows by $c$-affinity and homogeneity of $\tilde{V}$. Step 2.

Assume now that $w=\left(w_{1}, w_{2}\right)$ and $w^{\prime}=\left(w_{1}^{\prime}, w_{2}^{\prime}\right)$ are $\mathscr{D}$ such that $\tilde{V}(w)=$ $\tilde{V}\left(w^{\prime}\right), w_{1} \leq w_{2}, w_{1}^{\prime} \geq w_{2}^{\prime}$, and $\bar{w}=(t, t) \in \mathscr{D}$ be such that $\tilde{V}(\bar{w})=\tilde{V}(w)$. We assume, without loss of generality, $0<w_{1} \leq w_{2}^{\prime}$ (and thus, by axiom 8 and lemma $5, w_{2} \geq w_{2}^{\prime}$ ) and $0<w_{2}^{\prime}$.

By lemma $5, \tilde{V}\left(w_{1}, w_{2}\right)=\tilde{V}\left(w_{2}, w_{1}\right)$. Thus $\tilde{V}\left(w_{2}, w_{1}\right)=\tilde{V}\left(w_{1}^{\prime}, w_{2}^{\prime}\right)$. By the preceding argument, there exits $\theta \in[0,1]$ such that $w^{\prime}=\theta\left(w_{2}, w_{1}\right)+(1-\theta) \bar{w}$. Therefore, for all $\alpha \in[0,1]$ :

$\tilde{V}\left(\alpha w+(1-\alpha) w^{\prime}\right)=\tilde{V}\left(\alpha w+(1-\alpha)\left(\theta\left(w_{2}, w_{1}\right)+(1-\theta) \bar{w}\right)\right)$ 


$$
\begin{aligned}
= & \tilde{V}\left(\left(\alpha w+(1-\alpha) \theta\left(w_{2}, w_{2}\right)\right)+(1-\alpha)(1-\theta) \bar{w}\right) \\
= & (\alpha+(1-\alpha) \theta) \tilde{V}\left(\frac{\alpha}{\alpha+(1-\alpha) \theta} w+\frac{1-\alpha}{\alpha+(1-\alpha) \theta}\left(w_{2}, w_{1}\right)\right) \\
& +(1-\alpha)(1-\theta) \tilde{V}(\bar{w}), \text { by } c-\text { affinitiy and homogeneity of } \tilde{V} \\
\geq & (\alpha+(1-\alpha) \theta) \tilde{V}(w)+(1-\alpha)(1-\theta) \tilde{V}(\bar{w}), \text { by axiom } 9,
\end{aligned}
$$

and therefore $\tilde{V}\left(\alpha w+(1-\alpha) w^{\prime}\right) \geq \tilde{V}(w)$, the desired result.

Step 3.

It remains to deal with the case where $\tilde{V}(w) \neq \tilde{V}\left(w^{\prime}\right)$. Assume without loss of generality that $\tilde{V}(w)>\tilde{V}\left(w^{\prime}\right)$. Let $\mu=\tilde{V}(w)-\tilde{V}\left(w^{\prime}\right)$. Define $\tilde{w}=$ $w^{\prime}+(\mu, \mu)$. By c-affinity of $\tilde{V}$, we have $\tilde{V}(\tilde{w})=\tilde{V}\left(w^{\prime}\right)+\mu=\tilde{V}(w)$. Thus, for all $\alpha \in[0,1]$,

$$
\begin{aligned}
\tilde{V}(\alpha \tilde{w}+(1-\alpha) w) & \geq \alpha \tilde{V}(\tilde{w})+(1-\alpha) \tilde{V}(w), \text { by steps } 1 \text { and } 2 \\
& \geq \alpha \tilde{V}\left(w^{\prime}\right)+(1-\alpha) \tilde{V}(w)+\alpha \mu
\end{aligned}
$$

On the other hand,

$$
\begin{aligned}
\tilde{V}(\alpha \tilde{w}+(1-\alpha) w) & =\tilde{V}\left(\alpha\left(w^{\prime}+\mu\right)+(1-\alpha) w\right) \\
& =\tilde{V}\left(\alpha w^{\prime}+(1-\alpha) w\right)+\alpha \mu \text { by c-affinitiy of } \tilde{V} .
\end{aligned}
$$

Therefore $\tilde{V}\left(\alpha w^{\prime}+(1-\alpha) w\right) \geq \alpha \tilde{V}\left(w^{\prime}\right)+(1-\alpha) \tilde{V}(w)$, the desired result.

By Lemma 3, 4 and 6, $\tilde{V}$ is concave and homogeneous of degree 1, and c-affine. Therefore, by a classical result (see, e.g., the "Fundamental Lemma" in Chateauneuf (1991) and Lemma 3.5 in Gilboa and Schmeidler (1989)), there exists a unique closed and convex set $\Pi$ such that $\tilde{V}\left(w_{1}, w_{2}\right)=\min _{\pi \in \Pi} \pi(1) w_{1}+$ $\pi(2) w_{2}$. Furthermore, by lemma $5, \Pi$ is symmetric. Lemma 1 yields to Theorem 1.

\subsection{Proof of Proposition 1}

This proposition is in the vein of Theorem 3 in Gajdos, Tallon, and Vergnaud (2004) and Theorem 4 in Gajdos, Hayashi, Tallon, and Vergnaud (2008). For sake of exactness, we adapt the proof here.

It is straightforward to check that 2 implies 1. 
Conversely, suppose ad absurdum that $\succcurlyeq_{b}^{*}$ is more averse to imprecision than $\succcurlyeq_{a}^{*}$ but that there exists $p^{*} \in \varphi_{a}(P)$ such that $p \notin \varphi_{b}(P)$. Using a separation argument, there exists a function $\phi: \Omega \rightarrow \mathbb{R}$ such that $E_{p^{*}} \phi<$ $\min _{p \in \varphi_{b}(P)} E_{p} \phi$. Let $\bar{x}$ and $\underline{x}$ in $X$ be such that both $a$ and $b$ strictly prefer $\bar{x}$ to $\underline{x}$. Note that we can choose by normalization $u_{a}$ and $u_{b}$ so that $u_{a}(\bar{x})=$ $u_{b}(\bar{x})=1>u_{a}(\underline{x})=u_{b}(\underline{x})=0$. Since $\Omega$ is a finite set, there exist numbers $m>0$ and $\ell$, such that for all $\omega \in \Omega, m \phi(\omega)+\ell \in[0,1]$. Let $\alpha_{\omega}=m \phi(\omega)+\ell$, $\omega \in \Omega$. Let $f^{0} \in \mathscr{F}_{\bar{x}, \underline{x}}^{b}$ such that $f^{0}(\omega)=\alpha_{\omega} \delta_{\bar{x}}+\left(1-\alpha_{\omega}\right) \delta_{\underline{x}}$ for all $\omega \in \Omega$. Then, $E_{p^{*}} u\left(f^{0}\right)<\min _{p \in \varphi_{b}(P)} E_{p} u\left(f^{0}\right)$ which implies that $(f, P) \succ_{b}^{*}\left(f,\left\{p^{*}\right\}\right)$. However, since $p^{*} \in \varphi_{a}(P), E_{p^{*}} u\left(f^{0}\right) \geq \min _{p \in \varphi_{a}(P)} E_{p} u\left(f^{0}\right)$ which implies that $\left(f,\left\{p^{*}\right\}\right) \succcurlyeq_{a}^{*}(f, P)$ and thus yields a contradiction with $\succcurlyeq_{b}^{*}$ being more averse to imprecision than $\succcurlyeq_{a}^{*}$.

\subsection{Proof of Proposition 2}

Since $\Pi_{a}$ and $\Pi_{b}$ are symmetric, there exist $\alpha_{a}$ and $\alpha_{b}$ such that $\Pi_{a}=\{(1-$ $\left.\alpha_{a}\right)\left(\frac{1}{2}, \frac{1}{2}+\alpha_{a}(t, 1-t) \mid t \in[0,1]\right\}$ and $\Pi_{b}=\left\{\left(1-\alpha_{b}\right)\left(\frac{1}{2}, \frac{1}{2}+\alpha_{b}(t, 1-t) \mid t \in[0,1]\right\}\right.$. For all $f \in \mathscr{F}, P, Q \in \mathscr{P}, \alpha \in(0,1), i=a, b,(f, \alpha P+(1-\alpha) Q, \alpha P+(1-$ $\alpha) Q) \succcurlyeq_{i}(f, P, Q)$ iff:

$$
\min _{p \in \varphi_{i}(\alpha P+(1-\alpha) Q)} E_{p} u(f) \geq \min _{(\pi, 1-\pi) \in \Pi_{i}}\left(\pi \min _{p \in \varphi_{i}(P)} E_{p} u(f)+(1-\pi) \min _{p \in \varphi_{i}(Q)} E_{p} u(f)\right) .
$$

Since the $\varphi_{i}$ are linear, we then obtain:

$$
\begin{aligned}
\alpha \min _{p \in \varphi_{i}(P)} E_{p} u(f)+(1-\alpha) & \min _{p \in \varphi_{i}(Q)} E_{p} u(f) \\
\geq\left(\frac{1+\alpha_{i}}{2}\right) & \min \left(\min _{p \in \varphi_{i}(P)} E_{p} u(f), \min _{p \in \varphi_{i}(Q)} E_{p} u(f)\right) \\
& +\left(\frac{1-\alpha_{i}}{2}\right) \max \left(\min _{p \in \varphi_{i}(P)} E_{p} u(f), \min _{p \in \varphi_{i}(Q)} E_{p} u(f)\right)
\end{aligned}
$$

Furthermore, if both $a$ and $b$ prefer $(f, P)$ to $(f, Q)$, then $(f, \alpha P+(1-$ $\alpha) Q, \alpha P+(1-\alpha) Q) \succcurlyeq_{i}(f, P, Q)$ iff $\alpha \geq \frac{1-\alpha_{i}}{2}$.

Thus if $\succcurlyeq_{b}$ is more averse to conflict than $\succcurlyeq_{a}$, then $\frac{1-\alpha_{a}}{2} \geq \frac{1-\alpha_{b}}{2}$ and thus $\Pi_{a} \subseteq \Pi_{b}$. Conversely, if $\Pi_{a} \subseteq \Pi_{b}$, then $\succcurlyeq_{b}$ is more averse to conflict than $\succcurlyeq_{a}$. 


\subsection{Proof of Proposition 3}

Assume without loss of generality that $\Omega=\{1,2\}$. Let $\delta_{1}=(1,0), \delta_{2}=(0,1)$ and $\delta_{12}=\left\{p=\left(p_{1}, p_{2}\right) \in[0,1]^{2} \mid p_{1}+p_{2}=1\right\}$. Any set $P \in \mathscr{P}$ can be written in a unique way as: $P=\gamma_{1} \delta_{1}+\gamma_{2} \delta_{2}+\gamma_{3} \delta_{12}$, with $\gamma_{1}, \gamma_{2}, \gamma_{3} \geq 0$ such that $\gamma_{1}+\gamma_{2}+\gamma_{3}=1$.

For all $R=\gamma_{1} \delta_{1}+\gamma_{2} \delta_{2}+\gamma_{3} \delta_{12}$, and all $\theta \in[0,1]$, we have:

$$
\varphi(R)=\left(\gamma_{1}+\frac{(1-\theta) \gamma_{3}}{2}\right) \delta_{1}+\left(\gamma_{2}+\frac{(1-\theta) \gamma_{3}}{2}\right) \delta_{2}+\theta \gamma_{3} \delta_{12}
$$

Let $P=\lambda_{1} \delta_{1}+\lambda_{2} \delta_{2}+\lambda_{3} \delta_{12}, Q=\lambda_{1}^{\prime} \delta_{1}+\lambda_{2}^{\prime} \delta_{2}+\lambda_{3}^{\prime} \delta_{12}$ and $\alpha \in[0,1], \theta \in[0,1]$. Without any loss of generality, let us assume that $\lambda_{1}^{\prime}+\frac{1-\theta}{2} \lambda_{3}^{\prime} \geq \lambda_{1}+\frac{1-\theta}{2} \lambda_{3}$.

Consider a first case where $\theta=0$. Then

$$
\begin{aligned}
& \varphi(P)=\left(\lambda_{1}+\frac{\lambda_{3}}{2}\right) \delta_{1}+\left(\lambda_{2}+\frac{\lambda_{3}}{2}\right) \delta_{2}, \\
& \varphi(Q)=\left(\lambda_{1}^{\prime}+\frac{\lambda_{3}^{\prime}}{2}\right) \delta_{1}+\left(\lambda_{2}^{\prime}+\frac{\lambda_{3}^{\prime}}{2}\right) \delta_{2} .
\end{aligned}
$$

Simple computations show that

$$
\begin{aligned}
& \Pi \otimes\left(a \delta_{1}+(1-a) \delta_{2}, b \delta_{1}+(1-b) \delta_{2}\right) \\
= & \left(\frac{1+\alpha}{2} a+\frac{1-\alpha}{2} b\right) \delta_{1}+\left(\frac{1-\alpha}{2} a+\frac{1+\alpha}{2} b\right) \delta_{2}+(b-a) \alpha \delta_{12}
\end{aligned}
$$

if $a \leq b$.

If $R$ is such that $\varphi(R)=\Pi \otimes(\varphi(P), \varphi(Q))$, then we must have

$$
\begin{aligned}
\varphi(R) & =\left(\gamma_{1}+\frac{\gamma_{3}}{2}\right) \delta_{1}+\left(\gamma_{2}+\frac{\gamma_{3}}{2}\right) \delta_{2}=\Pi \otimes(\varphi(P), \varphi(Q)) \\
& =\left(\frac{1+\alpha}{2} a+\frac{1-\alpha}{2} b\right) \delta_{1}+\left(\frac{1-\alpha}{2} a+\frac{1+\alpha}{2} b\right) \delta_{2}+(b-a) \alpha \delta_{12}
\end{aligned}
$$

where $a=\lambda_{1}+\frac{\lambda_{3}}{2}$ and $b=\lambda_{1}^{\prime}+\frac{\lambda_{3}^{\prime}}{2}$.

Therefore, we must have $\alpha=0$ and thus $\theta \geq \alpha$.

Conversely, if $\theta=\alpha=0$, for $R=\Pi \otimes(P, Q), \varphi(R)=\Pi \otimes(\varphi(P), \varphi(Q))$ and thus for all $P, Q \in \mathscr{P}$, there exists $R \in \mathscr{P}$ such that $\Pi \otimes(\varphi(P), \varphi(Q))=\varphi(R)$.

Let suppose now that $\theta>0$. We show first that $(i)$ implies $(i i)$. Consider 
the case where $\lambda_{2}=1$ and $\lambda_{1}^{\prime}=1$. Then:

$$
\begin{aligned}
\Pi \otimes(\varphi(P), \varphi(Q)) & =\Pi \otimes\left(\delta_{2}, \delta_{1}\right) \\
& =\frac{1-\alpha}{2} \delta_{1}+\frac{1-\alpha}{2} \delta_{2}+\alpha \delta_{12} .
\end{aligned}
$$

If $R$ is such that $\varphi(R)=\Pi \otimes(\varphi(P), \varphi(Q))$, then we must have:

$$
\begin{aligned}
\varphi(R) & =\left(\gamma_{1}+\frac{(1-\theta) \gamma_{3}}{2}\right) \delta_{1}+\left(\gamma_{2}+\frac{(1-\theta) \gamma_{3}}{2}\right) \delta_{2}+\theta \gamma_{3} \delta_{12} \\
& =\Pi \otimes(\varphi(P), \varphi(Q))=\frac{1-\alpha}{2} \delta_{1}+\frac{1-\alpha}{2} \delta_{2}+\alpha \delta_{12} .
\end{aligned}
$$

Thus $\gamma_{3}=\frac{\alpha}{\theta}$ and for $\alpha>\theta, \gamma_{3}>1$ which yields a contradiction.

We now show that $(i i)$ implies $(i)$. Assume that $\theta \geq \alpha$ and consider two subcases.

Case where $\lambda_{2}^{\prime}+\frac{(1-\theta) \lambda_{3}^{\prime}}{2} \geq \lambda_{2}+\frac{(1-\theta) \lambda_{3}}{2}$ (it correponds to the case where $Q \subseteq P$ ).

Simple computations show that

$$
\begin{aligned}
\Pi \otimes\left(a_{1} \delta_{1}+\right. & \left.a_{2} \delta_{2}+\left(1-a_{1}-a_{2}\right) \delta_{12}, b_{1} \delta_{1}+b_{2} \delta_{2}+\left(1-b_{1}-b_{2}\right) \delta_{12}\right) \\
= & \left(\frac{1+\alpha}{2} a_{1}+\frac{1-\alpha}{2} b_{1}\right) \delta_{1}+\left(\frac{1+\alpha}{2} a_{2}+\frac{1-\alpha}{2} b_{2}\right) \delta_{2} \\
& \quad\left[\left(\frac{1+\alpha}{2}\right)\left(1-a_{1}-a_{2}\right)+\left(\frac{1-\alpha}{2}\right)\left(1-b_{1}-b_{2}\right)\right] \delta_{12}
\end{aligned}
$$

if $a_{1} \leq b_{1}$ and $a_{2} \leq b_{2}$.

If $R$ is such that $\varphi(R)=\Pi \otimes(\varphi(P), \varphi(Q))$, we must have

$$
\begin{gathered}
\varphi(R)=\left(\gamma_{1}+\frac{(1-\theta) \gamma_{3}}{2}\right) \delta_{1}+\left(\gamma_{2}+\frac{(1-\theta) \gamma_{3}}{2}\right) \delta_{2}+\theta \gamma_{3} \delta_{12} \\
=\Pi \otimes(\varphi(P), \varphi(Q)) \\
=\left(\frac{1+\alpha}{2} a_{1}+\frac{1-\alpha}{2} b_{1}\right) \delta_{1}+\left(\frac{1+\alpha}{2} a_{2}+\frac{1-\alpha}{2} b_{2}\right) \delta_{2} \\
+\left[\left(\frac{1+\alpha}{2}\right)\left(1-a_{1}-a_{2}\right)+\left(\frac{1-\alpha}{2}\right)\left(1-b_{1}-b_{2}\right)\right] \delta_{12}
\end{gathered}
$$

where $a_{1}=\lambda_{1}+\frac{1-\theta}{2} \lambda_{3}, a_{2}=\lambda_{2}+\frac{(1-\theta)}{2} \lambda_{3}, b_{1}=\lambda_{1}^{\prime}+\frac{1-\theta}{2} \lambda_{3}^{\prime}$ and $b_{2}=\lambda_{2}^{\prime}+\frac{(1-\theta)}{2} \lambda_{3}^{\prime}$. 
Therefore, we have:

$$
\begin{aligned}
\gamma_{1}+\frac{(1-\theta) \gamma_{3}}{2} & =\frac{1+\alpha}{2} a_{1}+\frac{1-\alpha}{2} b_{1} \\
\gamma_{2}+\frac{(1-\theta) \gamma_{3}}{2} & =\frac{1+\alpha}{2} a_{2}+\frac{1-\alpha}{2} b_{2} \\
\theta \gamma_{3} & =\left(\frac{1+\alpha}{2}\right)\left(1-a_{1}-a_{2}\right)+\left(\frac{1-\alpha}{2}\right)\left(1-b_{1}-b_{2}\right)
\end{aligned}
$$

which leads to

$$
\begin{aligned}
& \gamma_{1}=\frac{1+\alpha}{2} \lambda_{1}+\frac{1-\alpha}{2} \lambda_{1}^{\prime} \\
& \gamma_{2}=\frac{1+\alpha}{2} \lambda_{2}++\frac{1-\alpha}{2} \lambda_{2}^{\prime} \\
& \gamma_{3}=\left(\frac{1+\alpha}{2}\right) \lambda_{3}+\left(\frac{1-\alpha}{2}\right) \lambda_{3}^{\prime},
\end{aligned}
$$

which are three values between 0 and 1 . In fact, when $Q \subseteq P$, there always exists $R$ such that $\varphi(R)=\Pi \otimes(\varphi(P), \varphi(Q))$ as soon as $\theta>0$. $R$ is simply:

$$
R=\left(\frac{1+\alpha}{2}\right) P+\left(\frac{1-\alpha}{2}\right) Q
$$

Case where $\lambda_{2}+\frac{(1-\theta) \lambda_{3}}{2} \geq \lambda_{2}^{\prime}+\frac{(1-\theta) \lambda_{3}^{\prime}}{2}$.

Simple computation gives that

$$
\begin{aligned}
& \Pi \otimes\left(a_{1} \delta_{1}+\right.\left.a_{2} \delta_{2}+\left(1-a_{1}-a_{2}\right) \delta_{12}, b_{1} \delta_{1}+b_{2} \delta_{2}+\left(1-b_{1}-b_{2}\right) \delta_{12}\right) \\
&=\left(\frac{1+\alpha}{2} a_{1}+\frac{1-\alpha}{2} b_{1}\right) \delta_{1}+\left(\frac{1-\alpha}{2} a_{2}+\frac{1+\alpha}{2} b_{2}\right) \delta_{2} \\
& \quad+\left[\left(\frac{1+\alpha}{2}\right)\left(1-a_{1}-b_{2}\right)+\left(\frac{1-\alpha}{2}\right)\left(1-b_{1}-a_{2}\right)\right] \delta_{12}
\end{aligned}
$$

if $a_{1} \leq b_{1}$ and $a_{2} \geq b_{2}$.

If $R$ is such that $\varphi(R)=\Pi \otimes(\varphi(P), \varphi(Q))$, then we must have

$$
\begin{array}{r}
\varphi(R)=\left(\gamma_{1}+\frac{(1-\theta) \gamma_{3}}{2}\right) \delta_{1}+\left(\gamma_{2}+\frac{(1-\theta) \gamma_{3}}{2}\right) \delta_{2}+\theta \gamma_{3} \delta_{12} \\
=\Pi \otimes(\varphi(P), \varphi(Q)) \\
=\left(\frac{1+\alpha}{2} a_{1}+\frac{1-\alpha}{2} b_{1}\right) \delta_{1}+\left(\frac{1-\alpha}{2} a_{2}+\frac{1+\alpha}{2} b_{2}\right) \delta_{2}
\end{array}
$$




$$
+\left[\left(\frac{1+\alpha}{2}\right)\left(1-a_{1}-b_{2}\right)+\left(\frac{1-\alpha}{2}\right)\left(1-b_{1}-a_{2}\right)\right] \delta_{12}
$$

where $a_{1}=\lambda_{1}+\frac{1-\theta}{2} \lambda_{3}, a_{2}=\lambda_{2}+\frac{(1-\theta)}{2} \lambda_{3}, b_{1}=\lambda_{1}^{\prime}+\frac{1-\theta}{2} \lambda_{3}^{\prime}$ and $b_{2}=\lambda_{2}^{\prime}+\frac{(1-\theta)}{2} \lambda_{3}^{\prime}$.

To show that there exists $R$, we first show that we can find $\gamma_{3}$ such that $1 \geq \gamma_{3} \geq 0$ and such that

$$
\theta \gamma_{3}=\left(\frac{1+\alpha}{2}\right)\left(1-a_{1}-b_{2}\right)+\left(\frac{1-\alpha}{2}\right)\left(1-b_{1}-a_{2}\right) .
$$

Then we must have:

$$
\gamma_{3}=\frac{1}{2}\left[\lambda_{3}+\lambda_{3}^{\prime}+\frac{\alpha}{\theta}\left(\lambda_{1}^{\prime}-\lambda_{1}+\lambda_{2}-\lambda_{2}^{\prime}\right)\right]
$$

Since $\lambda_{1}^{\prime}+\frac{1-\theta}{2} \lambda_{3}^{\prime} \geq \lambda_{1}+\frac{1-\theta}{2} \lambda_{3}$ and $\lambda_{2}+\frac{(1-\theta) \lambda_{3}}{2} \geq \lambda_{2}^{\prime}+\frac{(1-\theta) \lambda_{3}^{\prime}}{2}$ we have:

$$
\begin{aligned}
& \lambda_{1}^{\prime}-\lambda_{1} \geq \frac{1-\theta}{2}\left(\lambda_{3}-\lambda_{3}^{\prime}\right) \\
& \lambda_{2}-\lambda_{2}^{\prime} \geq \frac{1-\theta}{2}\left(\lambda_{3}^{\prime}-\lambda_{3}\right)
\end{aligned}
$$

and thus

$$
\lambda_{1}^{\prime}-\lambda_{1}+\lambda_{2}-\lambda_{2}^{\prime} \geq 0
$$

Therefore $\gamma_{3} \geq 0$.

On the other hand, since $\theta \geq \alpha$,

$$
\gamma_{3} \leq \frac{1}{2}\left[\lambda_{3}+\lambda_{3}^{\prime}+\lambda_{1}^{\prime}-\lambda_{1}+\lambda_{2}-\lambda_{2}^{\prime}\right]=\left[\lambda_{2}+\lambda_{3}+\lambda_{1}^{\prime}+\lambda_{3}^{\prime}-1\right] \leq 1 .
$$

That means that we can always find a $\gamma_{3}$ that fits for the size of the probability interval $\Pi \otimes(\varphi(P), \varphi(Q))$.

It remains to be shown that we can find $\gamma_{1}$ and $\gamma_{2}$ that can adjust for the margins. Let $\chi$ be the size of the probability interval $\Pi \otimes(\varphi(P), \varphi(Q))$. Once $\chi$ is fixed, and thus $\gamma_{3}=\frac{\chi}{\theta}$, we can find $\gamma_{1}$ and $\gamma_{2}$ values such that $\varphi(R)=\tau \delta_{1}+(1-\chi-\tau) \delta_{2}+\chi \delta_{12}$ for any $\tau \in\left[\frac{(1-\theta)}{2} \frac{\chi}{\theta}, 1-\frac{\chi}{\theta}+\frac{(1-\theta)}{2} \frac{\chi}{\theta}\right]$.

On the other hand, the weight on $\delta_{1}$ in the decomposition of $\Pi \otimes(\varphi(P), \varphi(Q))$ is equal to $\frac{1+\alpha}{2}\left(\lambda_{1}+\frac{1-\theta}{2} \lambda_{3}\right)+\frac{1-\alpha}{2}\left(\lambda_{1}^{\prime}+\frac{1-\theta}{2} \lambda_{3}^{\prime}\right)$ with

$$
\chi=\frac{1}{2}\left[\theta\left(\lambda_{3}+\lambda_{3}^{\prime}\right)+\alpha\left(\lambda_{1}^{\prime}-\lambda_{1}+\lambda_{2}-\lambda_{2}^{\prime}\right)\right] .
$$


For $\chi$ fixed, to minimize $\tau^{\prime}=\frac{1+\alpha}{2}\left(\lambda_{1}+\frac{1-\theta}{2} \lambda_{3}\right)+\frac{1-\alpha}{2}\left(\lambda_{1}^{\prime}+\frac{1-\theta}{2} \lambda_{3}^{\prime}\right)$, we have to consider $\lambda_{1}=\lambda_{1}^{\prime}=0$. Then

$$
\chi=\frac{1}{2}\left[\theta\left(\lambda_{3}+\lambda_{3}^{\prime}\right)+\alpha\left(\lambda_{3}^{\prime}-\lambda_{3}\right)\right]
$$

while

$$
\begin{aligned}
\tau^{\prime} & =\frac{1+\alpha}{2} \frac{1-\theta}{2} \lambda_{3}+\frac{1-\alpha}{2} \frac{1-\theta}{2} \lambda_{3}^{\prime} \\
& =\frac{1-\theta}{2} \frac{1}{2}\left(\lambda_{3}+\lambda_{3}^{\prime}+\alpha\left(\lambda_{3}-\lambda_{3}^{\prime}\right)\right) \\
& =\frac{1-\theta}{2} \frac{1}{2} \frac{1}{\theta}\left(\theta\left(\lambda_{3}+\lambda_{3}^{\prime}\right)+\alpha\left(\lambda_{3}^{\prime}-\lambda_{3}\right)+\alpha\left(\theta\left(\lambda_{3}-\lambda_{3}^{\prime}\right)-\left(\lambda_{3}^{\prime}-\lambda_{3}\right)\right)\right) \\
& =\frac{(1-\theta)}{2} \frac{\chi}{\theta}+\frac{1-\theta}{2} \frac{1}{2} \frac{\alpha}{\theta}(1-\theta)\left(\lambda_{3}^{\prime}-\lambda_{3}\right) .
\end{aligned}
$$

Since we have also $\lambda_{1}^{\prime}+\frac{1-\theta}{2} \lambda_{3}^{\prime} \geq \lambda_{1}+\frac{1-\theta}{2} \lambda_{3}$, then $\tau^{\prime} \geq \frac{(1-\theta)}{2} \frac{\chi}{\theta}$ : for a fixed $\chi$, the lowest weight on $\delta_{1}$ that can be observed for $\Pi \otimes(\varphi(P), \varphi(Q))$ can be obtained by some $\gamma_{1}$ and $\gamma_{2}$. Using a similar proof, the same result holds for the lowest weight on $\delta_{2}$ that can be observed for $\Pi \otimes(\varphi(P), \varphi(Q))$, which means that the highest weight on $\delta_{1}$ that can be observed for $\Pi \otimes(\varphi(P), \varphi(Q))$ can also be obtained by some $\gamma_{1}$ and $\gamma_{2}$.

Therefore, there exists $R$ such that $\varphi(R)=\Pi \otimes(\varphi(P), \varphi(Q))$.

\subsection{Proof of Proposition 4}

Let $p, q \in \Delta(\{1,2\})$, with $p \neq q$. For all $\alpha, \theta \in[0,1]$, we have:

$$
\Pi \otimes(\varphi(\{p\}), \varphi(\{q\}))=\Pi \otimes(\{p\},\{q\})
$$

Thus $\varphi(\Pi \otimes(\{p\},\{q\}))=\Pi \otimes(\varphi(\{p\}), \varphi(\{q\}))$ iff $\varphi(\Pi \otimes(\{p\},\{q\}))=\Pi \otimes$ $(\{p\},\{q\})$, which implies $\theta=1$. Thus if $\Pi$ and $\varphi$ commute, it must be the case that $\theta=1$. The converse is trivial.

\subsection{Proof of Proposition 5}

We use the same notations as in the proof of Proposition 3. We have:

$$
\Pi \otimes\left(\varphi\left(\delta_{12}\right), \varphi\left(\delta_{1}\right)\right)=\Pi \otimes\left(\frac{1-\theta}{2} \delta_{1}+\frac{1-\theta}{2} \delta_{2}+\theta \delta_{12}, \delta_{1}\right)
$$




$$
\begin{aligned}
=\left(\frac{1+\alpha}{2}\right. & \left.\frac{1-\theta}{2}+\frac{1-\alpha}{2}\right) \delta_{1}+\left(\frac{1-\alpha}{2} \frac{1-\theta}{2}\right) \delta_{2} \\
& +\left[\left(\frac{1+\alpha}{2}\right)\left(1-\frac{1-\theta}{2}\right)-\left(\frac{1-\alpha}{2}\right)\left(\frac{1-\theta}{2}\right)\right] \delta_{12} .
\end{aligned}
$$

On the other hand:

$$
\varphi\left(\operatorname{co}\left(\delta_{12} \cup\left\{\delta_{1}\right\}\right)\right)=\varphi\left(\delta_{12}\right)=\frac{1-\theta}{2} \delta_{1}+\frac{1-\theta}{2} \delta_{2}+\theta \delta_{12} .
$$

Thus if $\Pi \otimes\left(\varphi\left(\delta_{12}\right), \varphi\left(\delta_{1}\right)\right)=\varphi\left(\operatorname{co}\left(\delta_{12} \cup\left\{\delta_{1}\right\}\right)\right)$, it implies that

$$
\frac{1-\alpha}{2} \frac{1-\theta}{2}=\frac{1-\theta}{2}
$$

and thus $\alpha=\theta=1$. Thus $(i)$ implies (ii). The converse is trivial. 


\section{References}

Cabantous, L. (2007): "Ambiguity aversion in the field of insurance: insurers' attitude to imprecise and conflicting probability estimates," Theory and Decision, 62, 219-240.

Chateauneuf, A. (1991): "On the use of capacities in modeling uncertainty aversion and risk aversion," Journal of Mathematical Economics, 20, 343369.

Crès, H., I. Gilboa, and N. Vieille (2009): "Aggregation of multiple prior opinions," working paper.

Gajdos, T., T. Hayashi, J.-M. Tallon, and J.-C. Vergnaud (2008): "Attitude toward imprecise information," Journal of Economic Theory, $140(1), 27-65$.

Gajdos, T., J.-M. Tallon, and J.-C. Vergnaud (2004): "Decision making with imprecise probabilistic information," Journal of Mathematical Economics, 40, 647-681.

- (2008): "Representation and aggregation of preferences under uncertainty," Journal of Economic Theory, 141(1), 68-99.

Genest, C., And J. Zidek (1986): "Combining Probability Distributions: A Critique and an Annotated Bibliography," Statistical Science, 1, 114-148.

Gilboa, I., D. Samet, and D. Schmeidler (2004): "Utilitarian Aggregation of Beliefs and Tastes," Journal of Political Economy, 112, 932-938.

GilboA, I., And D. Schmeidler (1989): "Maximin expected utility with a non-unique prior," Journal of Mathematical Economics, 18, 141-153.

Harsanyi, J. (1955): "Cardinal Welfare, Individualistic Ethics, and Interpersonal Comparisons if Utility," Journal of Political Economy, 63, 309-321.

MCCONWAY, K. (1981): "Marginalization and the linear opinion pools," Journal of the American Statistical Association, 76(374), 410-414.

Mongin, P. (1995): "Consistent Bayesian Aggregation," Journal of Economic Theory, 66, 313-351. 
Schmeidler, D. (1989): "Subjective Probability and Expected Utility Without Additivity," Econometrica, 57(3), 571-587.

Smithson, M. (1999): "Conflict aversion: preference for ambiguity vs conflict ins sources and evidence," Organizational Behavior and Human Decision Processes, 79, 179-198.

Stone, M. (1961): "The opinion pool," Annals of Mathematical Statistics, $32,1339-1342$. 